\title{
BMJ Open Analgesic use, pain and daytime sedation in people with and without dementia in aged care facilities: a cross-sectional, multisite, epidemiological study protocol
}

\author{
Edwin C K Tan, ${ }^{1}$ Renuka Visvanathan, ${ }^{2,3}$ Sarah N Hilmer, ${ }^{4,5}$ Agnes I Vitry, ${ }^{6}$ \\ Tara Quirke, ${ }^{7}$ Tina Emery, ${ }^{8}$ Leonie Robson, ${ }^{8}$ Terry Shortt, ${ }^{8}$ Simon Sheldrick, ${ }^{8}$ \\ Sunny (Soon Won) Lee, ${ }^{8}$ Robyn Clothier, ${ }^{3,8}$ Emily Reeve, ${ }^{4,5}$ Danijela Gnjidic, ${ }^{4,9}$ \\ Jenni llomäki, ${ }^{1}$ J Simon Bell ${ }^{1,5,6,10}$
}

To cite: Tan ECK, Visvanathan R, Hilmer SN, et al. Analgesic use, pain and daytime sedation in people with and without dementia in aged care facilities: a cross-sectional, multisite, epidemiological study protocol. BMJ Open 2014;4 e005757. doi:10.1136/ bmjopen-2014-005757

- Prepublication history for this paper is available online To view these files please visit the journal online (http://dx.doi.org/10.1136/ bmjopen-2014-005757)

Received 22 May 2014 Accepted 27 May 2014

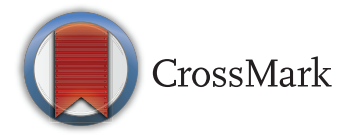

For numbered affiliations see end of article.

Correspondence to Professor J Simon Bell; simon.bell2@monash.edu

\section{ABSTRACT}

Introduction: People living with dementia may experience and express pain in different ways to people without dementia. People with dementia are typically prescribed fewer analgesics than people without dementia indicating a potential difference in how pain is identified and treated in these populations. The objectives of this study are to (1) investigate the prevalence of analgesic load, pain and daytime sedation in people with and without dementia in Australian residential aged care facilities (RACFs), and (2) investigate the clinical and diagnostic associations between analgesic load, pain and daytime sedation in people with and without dementia in Australian RACFs. Methods/analysis: This will be a cross-sectional study of 300 permanent residents of up to 10 low-level and high-level RACFs in South Australia with and without dementia. Trained study nurses will administer validated and dementia-specific assessments of selfreported and clinician-observed pain, sedation and other clinical and humanistic outcomes. Medicine-use data will be extracted directly from each resident's medication administration chart. Binary and multinominal logistic regression will be used to compute unadjusted and adjusted ORs and $95 \%$ Cls for factors associated with pain, analgesic load and daytime sedation. These factors will include dementia severity, behavioural and psychological symptoms, quality of life, resident satisfaction, attitudes towards medicines, activities of daily living and nutritional status.

Ethics and dissemination: Institutional ethics approval has been granted. The findings will be disseminated through public lectures, professional and scientific conferences and in peer-reviewed journal articles. The findings of this study will allow for a better understanding of the prevalence and factors associated with analgesic use, pain and other outcomes in residential care. The findings of this study will be used to inform the development and implementation of strategies to improve the quality of life of people with dementia.
Strengths and limitations of this study

A broad range of outcomes and risk factors will be assessed, thus allowing for the generation and exploration of a range of hypotheses. The additional prospective collection of hospitalisation and mortality data will enable an exploration of the effect of pain, analgesia and other covariates on the development of these outcomes.

- The findings of this research will enable a better understanding of the prescribing practices in aged care facilities and will assist in the development of targeted pain management services for people with dementia.

- The cross-sectional study design means it is difficult to make causal inferences about the data. Although the study will involve multiple aged care facilities, the generalisability may be limited. As with all observational studies, there will be the possibility of confounding.

\section{BACKGROUND}

A key objective when offering treatment to people with Alzheimer's disease (AD) and other dementias is to alleviate suffering and maintain quality of life. Pain is common among residents of residential aged care facilities (RACFs) with and without dementia. ${ }^{1}{ }^{2}$ The way that people with dementia experience and express pain is a topic of ongoing research. ${ }^{3}$

People with $\mathrm{AD}$ may communicate pain non-verbally via facial expressions, body movements and behavioural disturbances. These pain signs may go unrecognised and therefore not prompt clinicians to prescribe analgesic medicines. ${ }^{13}$ Most previous studies have reported lower overall use of analgesics 
in people with dementia compared with those without dementia and hypothesised that this may reflect untreated pain in these people. ${ }^{4-6}$ Untreated pain has been associated with increased use of healthcare and aggression. ${ }^{78}$ Use of analgesic medicines has been associated with higher quality of life in people with dementia. ${ }^{9}$ Pain-related behavioural disturbances may also be misinterpreted as behavioural and psychological symptoms of dementia leading to inappropriate prescription of psychotropic medicines which have been associated with impaired cognition, falls and fractures and increased risk of death. ${ }^{10} 11$

Clinicians working in RACFs are faced with the need to alleviate pain while minimising daytime sedation and prescription of psychotropic medicines. Medicine selection is also challenging as older people have a high prevalence of multimorbidity, frailty, polypharmacy and increased susceptibility to adverse drug events (ADEs). ${ }^{12-14}$ The lower prevalence of analgesic use among people with dementia may be partly linked to fear of ADEs (eg, excess sedation, falls and fractures, delirium and respiratory depression) as well as nonrecognition of pain. ${ }^{15-17}$ A cluster randomised controlled trial has demonstrated that a systematic approach to pain management, using a stepwise protocol for analgesic use, reduced agitation in aged care facility residents. ${ }^{18}$ This finding suggests that improved recognition and treatment of pain may be an effective strategy to reduce the unnecessary use of sedative and psychotropic medicines among residents of aged care facilities.

The aims of this study are to (1) investigate the prevalence of analgesic load, pain and daytime sedation in people with and without dementia in Australian RACFs and (2) investigate the clinical and diagnostic correlates of analgesic load, pain and daytime sedation in people with and without dementia in Australian RACFs.

\section{METHODS}

Design and setting

This will be a cross-sectional study among permanent residents of up to 10 low-level or high-level RACFs in South Australia. These RACFs will be located in metropolitan Adelaide (population 1.2 million) and $\mathrm{Mt}$ Gambier (population 25000). Adelaide is the state capital city and the fifth most populous city in Australia. Mt Gambier is a regional centre located approximately $450 \mathrm{~km}$ south of Adelaide. The aged care facilities will be approached to participate via one provider of aged care in South Australia.

\section{Consumer involvement}

The project will be conducted in accordance with Alzheimer's Australia guidelines for involving people living with dementia and their families in research (Alzheimer's Australia, 2013). The investigative team includes a member of the Alzheimer's Australia Consumer Dementia Research Network (TQ). The member of the Consumer Dementia Research Network has reviewed all project proposals from a consumer perspective, will participate on the project advisory committee and will assist in dissemination of the findings.

\section{Participants}

Permanent residents of low-level or high-level care RACFs will be invited to participate by a trained study nurse. This will include residents with and without dementia. Inclusion criteria will be age 65 years or older and ability to participate in structured assessments in English. Residents may be excluded at the discretion of RACF staff and their treating clinicians. Residents deemed by facility staff to be medically unstable (eg, experiencing delirium) or estimated by facility staff to have less than 3 months to live will be excluded from participation.

\section{Data collection}

Data will be collected using a standard data extraction form comprising a series of validated and widely used scales. The choice of scales was consistent with those included in the Dementia Management Outcomes Suite of the Australian Government Dementia Collaborative Research Centres. Data will be collected by experienced nurses employed by the participating aged care provider. Where possible, data will be collected by the same nurses at each RACF. Study nurses will undergo training in a centralised location in the standard administration of the study assessment tools. All nurses involved in the data collection will have undergone the mandatory police clearances for working with older people. The concordance between assessments performed by different study nurses will be established for an initial subsample of residents at one RACF. If required, each resident's general medical practitioner or community pharmacist may be contacted to provide additional data relevant to the study outcomes. For scales requiring input of a staff informant, staff informants will be required to have known the resident for at least 2 weeks. If during the data collection process a study nurse identifies a sign or symptom requiring medical attention, this will be brought to the attention of the care coordinator or senior registered nurse at the respective aged care facility.

\section{Primary outcomes}

Pain

Two validated pain scales suitable for use among people with dementia will be used. This will include a dementia-specific, clinician-administered observational scale (Pain Assessment in Advanced Dementia (PAINAD) Scale) and a resident self-report scale (FACES Pain Scale Revised (FPS-R)). The resident selfreport scale will be used in addition to the observational scale because observational scales may underestimate pain in people with dementia. ${ }^{6}{ }^{19}$ The scales will be administered when each resident is at rest. 
The PAINAD Scale is a five-item clinician-administered observational scale with domains related to breathing, negative vocalisation, facial expressions, body language and consolability. ${ }^{20}$ Possible scores range from 0 to 10 , with higher scores indicating more severe pain. The scale will be administered after observing the resident for $5 \mathrm{~min}$. The FPS-R is designed for people who are able to self-report but may be unable to use a traditional numeric rating scale. ${ }^{21}$ The scale consists of six faces that show how much pain or discomfort someone is feeling. The face on the left shows no pain whereas the face on the right shows the worst pain possible. The scale is scored from 0 'no pain' to 10 'worst pain possible'. Using the FPS has been demonstrated to be feasible and more easily understood than a traditional horizontal Visual Analogue Scale in people with a Mini-Mental State Examination (MMSE) score less than 11 and a Clinical Dementia Rating score of $3 .^{19}$

\section{Medicine use and analgesic load}

Medicine-use data from the previous $24 \mathrm{~h}$ will be extracted directly from each resident's medication administration chart by a study nurse. This will include prescription medicines, non-prescription medicines, vitamins and minerals and complementary and alternative medicines administered regularly and as required. The medicine name, strength, number of doses and whether it was administered will be recorded. This means that medicine-use data will represent each resident's actual use rather than their prescribed or intended pattern of use. Prescription and non-prescription medicines taken by the residents will be classified using the Anatomical Therapeutic Chemical (ATC) classification system recommended by the WHO. ${ }^{22}$

Analgesics will be defined as paracetamol (N02BE), non-steroidal anti-inflammatory drugs (ATC codes M01AB-H, M01AX01, M02AA, M02AC, N02BA) and opioids (N02A). Opioids will be subclassified as strong and weak opioids. Low-dose $(\leq 250 \mathrm{mg}$ /day) aspirin will not be classified as an analgesic because it is primarily used as an antiplatelet. Analgesic load will be calculated according to a previously published model developed in the USA. ${ }^{23}$ To calculate each resident's analgesic load, analgesic ratings will be assigned to each medicine taken by each resident on a regular or as-needed basis.

- Opioids for the treatment of moderate-to-severe pain (analgesic rating 9, eg, morphine, fentanyl).

- Opioids for the treatment of mild-to-moderate pain (analgesic rating 6, eg, tramadol).

- Non-opioid analgesics (analgesic rating 3, eg, ibuprofen, celecoxib).

- Adjuvant medicines (analgesic rating 1, eg, valproate, sertraline).

- Non-analgesic medicines (analgesic rating 0).

For each analgesic, a score will be derived by multiplying the daily dose by the analgesic rating and dividing by the average daily maintenance dose. The scores for each analgesic will be summed to compute each resident's analgesic load.

Sedative load resulting from each resident's exposure to medicines with sedative properties will be computed using a published model. ${ }^{24}$ This model considers medicines prescribed for sedation, medicines with sedation as a prominent side-effect and medicines with a sedating component.

\section{Daytime sedation}

Daytime sedation will be assessed using a clinicianadministered observational scale (Pasero Opioid-induced Sedation Scale (POSS)) and a resident self-report scale (Epworth Sleepiness Scale (ESS)). The POSS is a five-item clinician-rated scale. ${ }^{25}$ The reliability and validity of the POSS has been demonstrated for measuring sedation during administration of opioids. ${ }^{26}$ The ESS is a widely used eight-item resident self-report scale. Respondents are asked to rate their chance of falling asleep in eight different situations on a four-point scale. The ESS has been used among people with $\mathrm{AD},{ }^{27}$ and to assess the contribution of medicines to daytime sedation. ${ }^{28}$ The scale has also been successfully used previously in the hostel setting. ${ }^{29}$ Sleep quality will also be assessed using a previously validated six-item questionnaire. Three questions pertain to night-time sleep behaviour, including overall sleep quality, sleeping duration and restfulness. Other questions address alertness in the mornings and the frequency and duration of daytime napping. ${ }^{30}$

\section{Covariates and secondary outcomes Dementia severity}

The Dementia Severity Rating Scale (DSRS) will be used to assess the severity of each resident's dementia. This is a 12-item scale designed to be completed in consultation with a staff informant. ${ }^{31}$ It has been demonstrated to be a valid, reliable and sensitive measure of impairment associated with $\mathrm{AD} .{ }^{31}$ Enrolled or registered nurses will complete the scale.

\section{Behavioural and psychological symptoms}

Behavioural and psychological symptoms common in dementia will be assessed using the Neuropsychiatric Inventory Nursing Home version (NPI-NH). ${ }^{32}$ The NPI-NH contains the same 12 domains as the original NPI ${ }^{33}$ but has been reworded to allow for completion in consultation with staff informants who did not know the resident prior to the onset of illness. The NPI-NH has domains related to delusions, hallucinations, aggression/ agitation, depression/dysphoria, anxiety, elation/euphoria, apathy/indifference, disinhibition, irritability/lability and aberrant motor behaviour, sleep and night-time behaviour disorders and appetite and eating disorders.

\section{Quality of life}

Each resident's quality of life will be assessed by a registered or enrolled nurse using the staff informant version of the 15-item Quality of Life in Alzheimer's Disease 
Scale (QoL-AD). The 15-item version for use in RACFs is an adaption of the original 13-item version for use among community-dwelling people with dementia. ${ }^{34}$ Previous research suggests the QoL-AD scale has good validity and reliability when used for people with dementia with MMSE scores greater than $10 .^{35}$ In a recent study, the 15-item QoL-AD scale was successfully completed by $64 \%$ of residents with dementia in West Australian RACFs. ${ }^{36}$

\section{Attitudes towards medicine regimen}

Residents' attitudes towards medicines will be assessed using the 10-item Patients' Attitudes Towards Deprescribing (PATD) questionnaire. ${ }^{37}$ The PATD has satisfactory criterion validity and test-retest reliability when used in general adult samples. The PATD will be interviewer administered by the study nurse. The face validity of the PATD in people with dementia will be assessed prior to use.

\section{Resident satisfaction}

Resident satisfaction will be assessed using Hawthorne's revised 7-item Short Assessment of Patient Satisfaction (SAPS) ${ }^{38}$ Residents will be asked questions in relation to their overall care at the facilities, with an emphasis on interactions with nursing staff and carers.

\section{Other clinical factors}

Each resident's nutritional status will be assessed using the Mini Nutritional Assessment Short Form (MNA-SF) ${ }^{39}$ The MNA-SF has been validated as an assessment tool for evaluating malnutrition in elderly people. ${ }^{39}{ }^{40}$ Nutritional status has been associated with polypharmacy and is strongly predictive of poor prognosis in residents of aged care facilities. ${ }^{41}$ Activities of daily living will be assessed using the Katz Activities of Daily Living (ADL) scale. $^{42}$ This six-item scale includes domains related to bathing, dressing, toileting, transferring, continence and feeding. Diagnostic data will be extracted directly from each resident's electronic case notes and be used to compute a range of comorbidity indices including the Functional Comorbidity Index (FCI). ${ }^{43}$

\section{Hospitalisation and mortality}

RACF records will be used to determine the number, reasons for and duration of days in hospital over a 36-month period following the cross-sectional data collection. Data on deaths that occur over a 36-month period following the cross-sectional data collection will be obtained from the Consumer and Business Services: Births, Deaths and Marriages, South Australia. Hospitalisation and mortality data will be investigated because a previous study has suggested that potentially inappropriate medicine use defined using Beers criteria is associated with increased hospitalisation and mortality among residents of aged care facilities. ${ }^{44}$
Sample size and statistical analyses

Assuming a power of $80 \%$, a two-sided level of significance of 0.05 , dementia prevalence of $50 \%{ }^{45} 46$ and an analgesic prevalence of $60 \%,{ }^{46}$ a sample size of 258 participants would be required to detect a significant prevalence ratio (PR) of 0.7 in our primary outcome of analgesic use (OpenEpi V.2.3.1). To maintain adequate power in multivariate analyses, an approximate sample size of 5-20 cases is required per adjustment variable. For this reason, we will aim to recruit up to 300 residents with and without dementia to allow our analyses to be adjusted for a range of clinically important covariates. This sample size is consistent with similar cohort studies investigating quality of life and neuropsychiatric symptoms in people with dementia in Australia and internationally. ${ }^{36} 47$

Regression analyses will be performed to compute unadjusted and adjusted ORs and 95\% CIs for the association between pain, analgesic load and daytime sedation in people with $\mathrm{AD}$ and other dementias. The ORs will be transformed to PRs when outcomes are more common than $10 \% .{ }^{48}$ Multiple imputation will be used to impute missing covariate values where applicable. This has been demonstrated to be superior complete case analyses when data are missing at random. ${ }^{49}$ Analyses will be adjusted for age, sex, medicine use (eg, sedative load), diagnoses and other clinically relevant covariates. In addition to standard reporting in tables and figures, results will also be presented as threedimensional, geometric frameworks to provide a visual representation. ${ }^{50}$ Analyses will be undertaken using the Statistical Package for the Social Sciences (SPSS, V.17.0, Chicago, Illinois, USA).

\section{Ethical considerations}

A study nurse employed by the aged care provider will be responsible for inviting each resident to participate in the project. All potential resident participants will be provided with verbal and written information about the study. This will be carried out in accordance with the ethical principles for involving people with cognitive impairment in research studies that are outlined in the Australian National Statement for Ethical Conduct in Human Research. ${ }^{51}$ Consent to participate will be obtained from the residents if they have the capacity to consent. In cases where residents are unable to provide written informed consent themselves, consent to participate will be sought from their guardian, next of kin or significant other. Residents with an enduring power of guardianship will be identified by the participating aged care provider. In these cases, the study nurse will still explain to the resident, as much as possible, what participation will involve and consent will be sought from the guardian. All data will be treated confidentially and in accordance with Australian regulations. Data will be stored in a locked cabinet at the Centre for Medicine Use and Safety, Faculty of Pharmacy and Pharmaceutical Sciences, Monash University for a minimum of 5 years. 
The study will be conducted in accordance with the World Medical Association (WMA) Declaration of Helsinki.

\section{Dissemination of research findings}

The findings will be communicated to consumers and carers at one or more public lectures, with consumers and carers involved in the preparation and delivery of the lecture. The results will be presented at professional and scientific conferences. The results will also be disseminated in peer-reviewed scientific journal articles. All clinicians and RACFs that participate in the research will be provided with a copy of the final report, which will include a lay summary of the findings.

\section{DISCUSSION}

This study aims to improve pain management and, in turn, the quality of life of residents of RACFs with dementia. It will investigate outcomes that are important to consumers, and will involve close liaison with consumers, carers and clinicians throughout the study. The cross-sectional study design will enable the investigation of the prevalence of analgesic use, pain, sedation and other outcomes and the factors associated with these outcomes in residents of RACFs. Several different outcomes and risk factors will be assessed, thus allowing for the generation and exploration of a range of hypotheses. The additional prospective collection of hospitalisation and mortality data will enable an exploration of the effect of pain, analgesia and other covariates on the development of these outcomes. The findings of this research will enable a better understanding of the prescribing practices in RACFs and will assist in the development of targeted pain management services for people with dementia.

\section{Limitations}

The cross-sectional study design means it is difficult to make causal inferences about the data. Generalisability may be limited to the sampled population, and findings may not be applicable to all older people or other settings. Selection bias may occur, with volunteer bias being a potential limitation. Residents and families choosing to participate in the study may tend to be those who are healthier, have less cognitive impairment or are more involved in their pain management. These issues will be minimised by ensuring a census sample of eligible residents is recruited from each facility where possible. Additionally, demographics of the participants will be compared with all residents of the RACF to assess representativeness of the sample. Close engagement and monitoring by the research team will ensure that recruitment and data collection protocols are implemented consistently.

\section{CONCLUSION}

This study will investigate the association between medication use and clinical characteristics in residents with and without dementia in RACFs. The findings of this study will allow for a better understanding of the prevalence and risk factors associated with analgesic use, pain, sedation and other outcomes in aged care settings. This will help guide the development and implementation of strategies to improve the quality of life of people with dementia.

\section{Author affiliations}

${ }^{1}$ Faculty of Pharmacy and Pharmaceutical Sciences, Centre for Medicine Use and Safety, Monash University, Melbourne, Australia

${ }^{2}$ Discipline of Medicine, University of Adelaide, Adelaide, Australia

${ }^{3}$ Aged and Extended Care Services, The Queen Elizabeth Hospital and Adelaide Geriatrics Training and Research with Aged Care (GTRAC) Centre, School of Medicine, University of Adelaide, Adelaide, Australia

${ }^{4}$ Departments of Clinical Pharmacology and Aged Care, Kolling Institute of Medical Research, Sydney Medical School, Royal North Shore Hospital, The University of Sydney, Sydney, Australia

${ }^{5}$ Cognitive Decline Partnership Centre, Hornsby Ku-ring-gai Hospital, Hornsby, Australia

${ }^{6}$ Sansom Institute, School of Pharmacy and Medical Sciences, University of South Australia, Adelaide, Australia

${ }^{7}$ Consumer Dementia Research Network, Alzheimer's Australia, Scullin, Australia

${ }^{8}$ Resthaven Limited, Adelaide, South Australia

${ }^{9}$ Faculty of Pharmacy, University of Sydney, Sydney, Australia

${ }^{10}$ Kuopio Research Centre of Geriatric Care, University of Eastern Finland, Kuopio, Finland

Acknowledgements The authors would like to thank the Resthaven management team, research nurses, facility staff and participants for their valuable support and cooperation.

Contributors JSB, RV, SNH and AIV conceived the study and obtained grant funding. JSB, ECKT, RV, SNH, AIV and TQ participated in the design of the study protocol. All authors critically reviewed the study protocol. TE, LR, TS, SS, SL and RC assisted in the development and implementation of the study. Data will be collated and analysed by JSB, ECKT, RV, ER, DG and JI. JSB and ECKT drafted the manuscript. All authors read and approved the final manuscript.

Funding This work is supported by the Alzheimer's Australia Dementia Research Foundation via the Resthaven Incorporated Dementia Research Award, with additional funding provided by Resthaven Incorporated.

Competing interests DG is supported by a National Health and Medical Research Council Early Career Fellowship.

\section{Patient consent Obtained.}

Ethics approval The Royal Australian College of General Practitioners Nationa Research and Evaluation Ethics Committee and the Monash University Human Research Ethics Committee.

Provenance and peer review Not commissioned; internally peer reviewed.

Data sharing statement The majority of data collected will be published. Any unpublished, de-identified data will be made available to interested persons on request.

Open Access This is an Open Access article distributed in accordance with the Creative Commons Attribution Non Commercial (CC BY-NC 4.0) license, which permits others to distribute, remix, adapt, build upon this work noncommercially, and license their derivative works on different terms, provided the original work is properly cited and the use is non-commercial. See: http:// creativecommons.org/licenses/by-nc/4.0/ 


\section{REFERENCES}

1. Herr K. Pain assessment strategies in older patients. J Pain 2011;12:S3-13.

2. Piazza Research and Alzheimer's Australia. End of life care for people with dementia. Canberra: Alzheimer's Australia, 2014.

3. Scherder E, Herr K, Pickering G, et al. Pain in dementia. Pain 2009;145:276-8.

4. Scherder EJ, Bouma A. Is decreased use of analgesics in Alzheimer disease due to a change in the affective component of pain? Alzheimer Dis Assoc Disord 1997;11:171-4.

5. Schmader KE, Hanlon JT, Fillenbaum GG, et al. Medication use patterns among demented, cognitively impaired and cognitively intact community-dwelling elderly people. Age Ageing 1998;27:493-501.

6. Tan ECK, Jokanovic N, Koponen MP, et al. Analgesic use and pain in people with and without dementia or cognitive impairment in aged care facilities: a systematic review and meta-analysis. Curr Clin Pharmacol 2014; in press.

7. Kunik ME, Cully JA, Snow AL, et al. Treatable comorbid conditions and use of VA health care services among patients with dementia. Psychiatr Serv 2005;56:70-5.

8. Kunik ME, Snow AL, Davila JA, et al. Causes of aggressive behavior in patients with dementia. J Clin Psychiatry 2010;71:1145-52.

9. Cordner Z, Blass DM, Rabins PV, et al. Quality of life in nursing home residents with advanced dementia. J Am Geriatr Soc 2010;58:2394-400.

10. Ballard C, Hanney ML, Theodoulou M, et al. The dementia antipsychotic withdrawal trial (DART-AD): long-term follow-up of a randomised placebo-controlled trial. Lancet Neurol 2009;8:151-7.

11. Hartikainen S, Lonnroos E, Louhivuori K. Medication as a risk factor for falls: critical systematic review. J Gerontol A Biol Sci Med Sci 2007;62:1172-81.

12. Mitchell SJ, Hilmer SN, McLachlan AJ. Clinical pharmacology of analgesics in old age and frailty. Rev Clin Gerontol 2009;19: 103-18.

13. McLachlan AJ, Hilmer SN, Le Couteur DG. Variability in response to medicines in older people: phenotypic and genotypic factors. Clin Pharmacol Ther 2009;85:431-3.

14. Hilmer SN, Gnjidic D. The effects of polypharmacy in older adults. Clin Pharmacol Ther 2009;85:86-8.

15. Bell JS, Laitinen ML, Lavikainen $P$, et al. Use of strong opioids among community-dwelling persons with and without Alzheimer's disease in Finland. Pain 2011;152:543-7.

16. Murnion BP, Gnjidic D, Hilmer SN. Prescription and administration of opioids to hospital in-patients, and barriers to effective use. Pain Med 2010;11:58-66.

17. Schneider LS, Dagerman KS, Insel P. Risk of death with atypical antipsychotic drug treatment for dementia: meta-analysis of randomized placebo-controlled trials. JAMA 2005;294:1934-43.

18. Husebo BS, Ballard C, Sandvik R, et al. Efficacy of treating pain to reduce behavioural disturbances in residents of nursing homes with dementia: cluster randomised clinical trial. BMJ 2011;343:d4065.

19. Pautex S, Michon A, Guedira M, et al. Pain in severe dementia: self-assessment or observational scales? J Am Geriatr Soc 2006;54:1040-5

20. Warden V, Hurley AC, Volicer L. Development and psychometric evaluation of the Pain Assessment in Advanced Dementia (PAINAD) scale. J Am Med Dir Assoc 2003;4:9-15.

21. International Association for the Study of Pain. Faces Pain ScaleRevised. 2012. http://www.iasp-pain.org/Education/Content.aspx? ItemNumber $=1519$

22. The Anatomical Therapeutic Chemical Classification System. http://www.whocc.no/atc_ddd_index/

23. Sloane $\mathrm{P}$, Ivey $\mathrm{J}$, Roth $\mathrm{M}$, et al. Accounting for the sedative and analgesic effects of medication changes during patient participation in clinical research studies: measurement development and application to a sample of institutionalized geriatric patients. Contemp Clin Trials 2008;29:140-8.

24. Taipale HT, Bell JS, Soini H, et al. Sedative load and mortality among residents of long-term care facilities: a prospective cohort study. Drugs Aging 2009;26:871-81.

25. Pasero C, McCaffery M. Monitoring sedation. Am J Nurs 2002;102:67-9.

26. Nisbet AT, Mooney-Cotter F. Comparison of selected sedation scales for reporting opioid-induced sedation assessment. Pain Manag Nurs 2009;10:154-64.
27. Lee JH, Bliwise DL, Ansari FP, et al. Daytime sleepiness and functional impairment in Alzheimer disease. Am J Geriatr Psychiatry 2007;15:620-6.

28. O'Suilleabhain PE, Dewey RB Jr. Contributions of dopaminergic drugs and disease severity to daytime sleepiness in Parkinson disease. Arch Neurol 2002;59:986-9.

29. Latimer Hill E, Cumming RG, Lewis R, et al. Sleep disturbances and falls in older people. J Gerontol A Biol Sci Med Sci 2007;62:62-6.

30. St George RJ, Delbaere K, Williams P, et al. Sleep quality and falls in older people living in self- and assisted-care villages. Gerontology 2009;55:162-8.

31. Clark CM, Ewbank DC. Performance of the dementia severity rating scale: a caregiver questionnaire for rating severity in Alzheimer disease. Alzheimer Dis Assoc Disord 1996;10:31-9.

32. Wood S, Cummings JL, Hsu MA, et al. The use of the neuropsychiatric inventory in nursing home residents. Characterization and measurement. Am J Geriatr Psychiatry 2000;8:75-83.

33. Cummings JL, Mega M, Gray K, et al. The neuropsychiatric inventory: comprehensive assessment of psychopathology in dementia. Neurology 1994;44:2308-14.

34. Edelman P, Fulton BR, Kuhn D, et al. A comparison of three methods of measuring dementia-specific quality of life: perspectives of residents, staff, and observers. Gerontologist 2005;45(Spec No 1):27-36.

35. Logsdon RG, Gibbons LE, McCurry SM, et al. Assessing quality of life in older adults with cognitive impairment. Psychosom Med 2002;64:510-19.

36. Beer C, Flicker L, Horner B, et al. Factors associated with self and informant ratings of the quality of life of people with dementia living in care facilities: a cross sectional study. PLOS ONE 2010;5:e15621.

37. Reeve E, Shakib S, Hendrix I, et al. Development and validation of the patients' attitudes towards deprescribing (PATD) questionnaire. Int J Clin Pharm 2013;35:51-6.

38. Dementia Outcomes Measurement Suite. http://www. dementia-assessment.com.au/symptoms/

39. Guigoz Y, Lauque S, Vellas BJ. Identifying the elderly at risk for malnutrition. The Mini Nutritional Assessment. Clin Geriatr Med 2002;18:737-57.

40. Kaiser MJ, Bauer JM, Ramsch C, et al. Validation of the Mini Nutritional Assessment short-form (MNA-SF): a practical tool for identification of nutritional status. J Nutr Health Aging 2009;13:782-8.

41. Jyrkka J, Mursu J, Enlund $\mathrm{H}$, et al. Polypharmacy and nutritional status in elderly people. Curr Opin Clin Nutr Metab Care 2012;15:1-6.

42. Katz S, Ford AB, Moskowitz RW, et al. Studies of illness in the aged The index of ADL: a standardized measure of biological and psychosocial function. JAMA 1963;185:914-19.

43. Groll DL, To T, Bombardier C, et al. The development of a comorbidity index with physical function as the outcome. J Clin Epidemiol 2005;58:595-602.

44. Lau DT, Kasper JD, Potter DE, et al. Hospitalization and death associated with potentially inappropriate medication prescriptions among elderly nursing home residents. Arch Intern Med 2005;165:68-74.

45. Somers M, Rose E, Simmonds D, et al. Quality use of medicines in residential aged care. Aust Fam Physician 2010;39:413-16.

46. Stafford AC, Alswayan MS, Tenni PC. Inappropriate prescribing in older residents of Australian care homes. J Clin Pharm Ther 2011;36:33-44

47. Karttunen K, Karppi $P$, Hiltunen A, et al. Neuropsychiatric symptoms and quality of life in patients with very mild and mild Alzheimer's disease. Int J Geriatr Psychiatry 2011;26:473-82.

48. Zhang J, Yu KF. What's the relative risk? A method of correcting the odds ratio in cohort studies of common outcomes. JAMA 1998;280:1690-1

49. Janssen KJ, Donders AR, Harrell FE Jr, et al. Missing covariate data in medical research: to impute is better than to ignore. J Clin Epidemiol 2010;63:721-7.

50. Gnjidic D, Hilmer SN, Blyth FM, et al. High-risk prescribing and incidence of frailty among older community-dwelling men. Clin Pharmacol Ther 2012;91:521-8.

51. National Statement for Ethical Conduct in Human Research. http:// www.nhmrc.gov.au/_files_nhmrc/publications/attachments/e72 national statement march 2014 140331.pdf 